\title{
Hepatoma-derived growth factor predicts unfavorable prognosis of epithelial ovarian cancer
}

This article was published in the following Dove Press journal:

OncoTargets and Therapy

13 August 2015

Number of times this article has been viewed

Xue-jun Liu'

Wen-lian Liu'

Fang-mei Yang'

Xiao-qing Yang ${ }^{2}$

Xiao-fei Lu ${ }^{3}$

'Department of Obstetrics, Linyi Hospital Affiliated to Shandong University, Linyi City, ${ }^{2}$ Department of Pathology, Qianfoshan Hospital Affiliated to Shandong University, Jinan City, ${ }^{3}$ Department of General Surgery, Jinan Central Hospital Affiliated to Shandong University, Jinan City, People's Republic of China
Correspondence: Xiaofei Lu Department of Pathology, Jinan Central Hospital Affiliated to Shandong University, 105 Jiefang Road, Jinan 2500 I3, People's Republic of China $\mathrm{Tel}+8613854198278$

Email sduly2015@163.com
Aim: To evaluate the expression and clinical significance of hepatoma-derived growth factor (HDGF) in epithelial ovarian cancer (EOC).

Background: Recent studies have demonstrated that HDGF overexpression correlates to the progression and poor prognosis in several kinds of cancers. However, the clinical significance and prognostic value of HDGF in EOC have not been investigated.

Methods: Expression of HDGF was visualized by immunohistology and then the cohort was divided into higher- and lower-expression groups. The correlation between HDGF and clinicopathologic factors was analyzed by $\chi^{2}$ test. The prognostic value of HDGF was assessed by univariate analysis with Kaplan-Meier method, and by multivariate analysis with Cox-regression model. With experiments in vitro, HDGF expression in ovarian cancer cell lines was detected by immunoblotting.

Results: Higher HDGF expression rate was $52.76 \%$ in EOC. HDGF expression was significantly associated with lymphatic metastasis $(P=0.006)$. Higher HDGF expression was closely correlated to poorer 5-year overall survival rate with univariate analysis $(P=0.003)$, and was identified as an independent prognostic factor with multivariate analysis $(P=0.007)$. With experiments in vitro, HDGF was proved to exist in all ovarian cancer cell lines with different expression levels.

Conclusion: HDGF expression correlates to unfavorable prognosis and can be considered as an independent prognostic factor, indicating that HDGF may be a promising potential molecular drug target.

Keywords: biomarker, HDGF, knockdown, invasion

\section{Introduction}

Ovarian cancer is the most lethal gynecological malignancy and the fourth most frequent cancer in women. ${ }^{1}$ The morbidity of ovarian cancer is increasing worldwide. Approximately 160,000 people died from ovarian cancer in 2010, up from 113,000 in $1990 .^{2}$ The high lethal rate of ovarian cancer partly results from its silent symptoms and rapid metastasis in early stage. ${ }^{3}$ Approximately $70 \%$ of patients finally diagnosed with ovarian cancer present as stage III or IV. ${ }^{4}$ Later warning symptoms mainly include bloating, pelvic pain, and abdominal swelling. The most vulnerable organs to ovarian cancer invasion include lung, liver, or lymph nodes, usually via direct planting, local invasion, and lymphatic metastasis. ${ }^{1}$ The histologies of ovary cancer include epithelial ovarian cancer (EOC), malignant germ cell carcinoma, etc. ${ }^{5}$ As with the majority of ovarian cancers, EOCs consist of several histologic subtypes including serous, endometrioid, clear cell, and mucinous adenocarcinomas. ${ }^{6}$

Approximately, $70 \%$ of patients with ovarian cancer show clinical response, thanks to the development of surgery and adjacent therapies, including chemotherapy and radiotherapy. ${ }^{7}$ However, the majority of patients will experience relapse. ${ }^{1}$ Targeted therapy is one effective method to reduce the relapse of ovarian cancer, which may 
prolong the survival time and promote the life quality of ovarian cancer patients, just like what Herceptin does to breast cancer patients. The finding of effective targeted therapy depends on the discovery of effective tumor biomarkers and molecular targets. However, the discovery of new and effective biomarkers of ovarian cancer yielded little improvement in the past decades.

Hepatoma-derived growth factor (HDGF) is an acidic heparin-binding protein originally purified from cultured media of the human hepatoma cell line $\mathrm{HuH}-7,{ }^{8}$ which is widely expressed in human tissues including kidney, cardiovascular system, liver, intestine, and brain. HDGF is a secreted growth factor and could promote cellular processes like proliferation, differentiation, and migration of cells. ${ }^{9}$ The association between HDGF and cancer has been reported more and more recently. Abnormal overexpression and excessive function of HDGF have been revealed in several cancers including hepatoma, cholangiocarcinoma, gastrointestinal stromal tumors, pancreatic cancer, and gastric carcinoma. ${ }^{10-12}$ The underlying mechanism may be involved in HDGF promoting cancer cell growth via translocation to the nucleus, but the exact mechanism is still unclear. ${ }^{13}$ However, the significance of HDGF in ovarian cancer is still unrevealed. In our study, we detected the expression of HDGF in samples of 85 ovarian cancers, then analyzed the correlation between HDGF expression and clinicopathologic parameters and overall survival rates. Furthermore, we identified HDGF as an independent prognostic factor with multivariate analysis. To explore the reason why HDGF overexpression leads to unfavorable prognosis, we further performed experiments in vitro to study HDGF's influence on EOC cell invasion by in vitro study.

\section{Patients and methods Cell culture and agents}

The ovarian cancer cell lines SK-OV-3, HO9810, HO8910PM, and OVCAR3 were bought from Cell Bank of the Chinese Academy of Sciences (Shanghai, People's Republic of China). Ovarian cancer cell line OAW-42 was obtained from Sigma-Aldrich Co. (St Louis, MO, USA). SK-OV-3 and OAW-42 were cultured in McCOY's 5A medium (SigmaAldrich Co.), while HO9810, HO8910PM, and OVCAR3 were cultured in RPMI-1640 medium, supplemented with $10 \%$ fetal bovine serum, $100 \mathrm{U} / \mathrm{mL}$ penicillin, and $100 \mu \mathrm{g} / \mathrm{mL}$ streptomycin, under $5 \% \mathrm{CO}_{2}$ in a humidified incubator at $37^{\circ} \mathrm{C}$. The anti-HDGF(N-15) and anti-actin(I-19) antibodies were purchased from Santa Cruz Biotechnology Inc. (Dallas, TX, USA). The HDGF enzyme-linked immunosorbent assay
(ELISA) kit was obtained from Antibodies-online Company (Aachen, Germany).

\section{Patients and tissue samples}

The primary cohort contained 134 patients who were diagnosed as suffering with EOC, underwent surgical operation, and took standard post-operational adjustment therapy from 2004 to 2010. The validation cohort comprised 85 patients and was selected from the primary cohort according to the criteria as follows: 1) available tissue samples and medical records; 2) post-operational survival time is more than 3 months; and 3 ) available for follow-ups. The median follow-up time of the validation cohort was 27.1 months (range: 4-101 months). All of the ovarian cancer samples were obtained from the Pathology Department, with prior patient consent and approval of the Institutional Clinical Ethics Review Board. All specimens were obtained from the Pathology Department with prior patient consent and approval of the Institutional Clinical Ethics Review Board, and handled anonymously according to the ethical and legal standards. Clinical stage of epithelial cancer followed the International Federation of Gynecology and Obstetrics (FIGO) stage system.

\section{Immunohistochemistry and evaluation}

Immunohistochemical (IHC) staining was performed by the streptavidin-peroxidase complex method. In brief, slides with samples were first deparaffinized and rehydrated with xylene and graded alcohol, then incubated in $3 \%$ hydrogen peroxide for 10 minutes for endogenous peroxidase inactivation. For better staining effect, the optimal antigen retrieval was recommended, which was soaking in citrate buffer ( $\mathrm{pH}$ 6.0) for 10 minutes. After blocking in 5\% bovine serum albumin for 1 hour, samples were incubated in primary antibody at 1:100 dilution at $4{ }^{\circ} \mathrm{C}$ overnight, followed by incubation in secondary antibodies, streptavidin-peroxidase complex reagent, and 3,3'-diaminobenzidine solution, respectively. Every stained section was blindly evaluated by two pathologists unaware of the clinical data. Samples for which consensus could not be reached were reevaluated by a third observer. The standard of IHC score was as per a previous study. ${ }^{14}$ The score was quantified by the calculation of software Image-Pro Plus, and the mean value of IHC scores of all samples was set as the cutoff, which divided the validation cohort into higherexpression and lower-expression groups.

\section{Immunoblotting assay}

HDGF expression in ovarian cancer cell lines was evaluated and visualized by immunoblotting. Briefly, the total protein of 
cells, including the protein from nuclear and cytoplasm, was first extracted according to the manual of Total Cellular Protein Kit (catalogue number BSP003; Sangon Company). Concentration of protein from these cell lines was detected by BCA detection kit (P0010S; Beyotime Cooperation). Equal amounts of $20 \mu \mathrm{g}$ total protein was electrophoresed with sodium dodecyl sulfate-polyacrylamide gel electrophoresis gel, then transferred to polyvinylidene fluoride membrane (PALL Company, USA), and incubated in primary antibody $(1: 1,000)$ overnight at $4^{\circ} \mathrm{C}$. The polyvinylidene fluoride membrane was finally incubated with corresponding secondary antibody, labeled with horseradish peroxidase for 2 hours at $37^{\circ} \mathrm{C}$, and visualized by ECL (EMD Millipore, Billerica, MA, USA).

\section{Matrigel transwell assay}

The ability of tumor invasion was evaluated by Matrigel transwell assay with $8 \mu \mathrm{m}$ pore Matrigel-coated transwells (BD Biosciences, San Jose, CA, USA). Briefly, cells were passaged into the Matrigel invasion chambers and cultured for 6 hours for adherence. Then, medium starvation was carried out in serum-free medium for 6 hours, followed by incubation in $10 \mathrm{ng} / \mathrm{mL}$ HDGF in the upper chamber. After incubating for 24 hours, cells were fixed with $4 \%$ paraformaldehyde for 30 minutes and stained with $0.05 \%$ gentian violet for 1 hour. Cells on the upper surface were removed with a cotton swab and the cell number on the lower surface was counted from ten random fields. The cell number of the control group was set as a baseline and fold change was calculated by ratio to the control group. In one independent assay, duplication of every tested group was performed for transwell assay, and the analyzed data were from three different experiments. Statistical significance was evaluated by Student's $t$-test.

\section{HDGF detection by ELISA}

HDGF ELISA kit was used to detect the HDGF concentration in medium of EOC cell line according to the manual of the kit. Briefly, to detect secreted HDGF, tested samples were made after cell medium collection, centrifugation at 2,000 rpm, and filtration with $0.22 \mu \mathrm{m}$ filter. After incubating for 2 hours, samples were added to $100 \mu \mathrm{L}$ Detection Reagent A and incubated for 1 hour, subsequently washed with PBS, and incubated in substrate solution for 15 minutes. Finally, optical density at $450 \mathrm{~nm}$ was read after $50 \mu \mathrm{L}$ stop solution was added and mixed.

\section{RNA knockdown and transfection}

HDGF knockdown was accomplished by oligo-siRNA designed as per the previous study. ${ }^{15}$ The sequence of siRNA was 5'-GCCAUGUCUUCUCCCUGGA and 5'-UCCAG
GGAGAAGACAUGGC. Control siRNA was also ordered from Thermo Fisher Scientific (Waltham, MA, USA). Transfection of siRNA was realized by Lipofectamine 2000 reagent (Thermo Fisher Scientific) according to the manual.

\section{Statistical analysis}

All the data were analyzed by SPSS 17.0 software (SPSS Inc., Chicago, IL, USA). The correlation between HDGF expression and other clinicopathologic parameters was evaluated by $\chi^{2}$ test or Fisher's exact test. The association of HDGF with overall survival rate was analyzed by KaplanMeier method, and the difference in survival curves was evaluated with a log-rank test. The independent prognostic factors were finally identified with Cox proportional hazards regression model. $P<0.05$ was considered as statistically significant.

\section{Results HDGF expression in EOC}

In our experiments, HDGF was mainly observed in the nucleus of EOC (Figure 1A and B), which could be explained by the fact that HDGF functions as a transcription factor. According to the IHC score criteria, the HDGF expression was divided into higher-expression and lower-expression groups by the cutoff. The basic characteristics of patients with EOC are summarized in Table 1. The percentage of higher HDGF expression is $52.76 \%(44 / 83)$.

\section{Correlation between HDGF and clinicopathologic parameters}

To evaluate the comprehensive significance of HDGF in EOC, we further analyzed the correlation between HDGF expression and other clinicopathologic factors, including FIGO stage, pathologic grade, histology, and lymph node metastasis (Table 2). Higher HDGF expression was proved to be significantly associated with lymph node metastasis $(P=0.006)$. Cases with higher HDGF expression appeared to have higher probability of lymph metastasis, indicating that HDGF may play an essential role in the process of lymph invasion.

\section{Association of HDGF with overall survival rate}

The prognostic values of all the observed clinicopathologic parameters were evaluated by univariate analysis with the Kaplan-Meier method (Table 3). The 5-year overall survival rate of HGDF in higher- and lower-expression groups were $21.4 \%$ and $42.1 \%$, respectively (Figure $2 \mathrm{~A}$ ). HDGF 


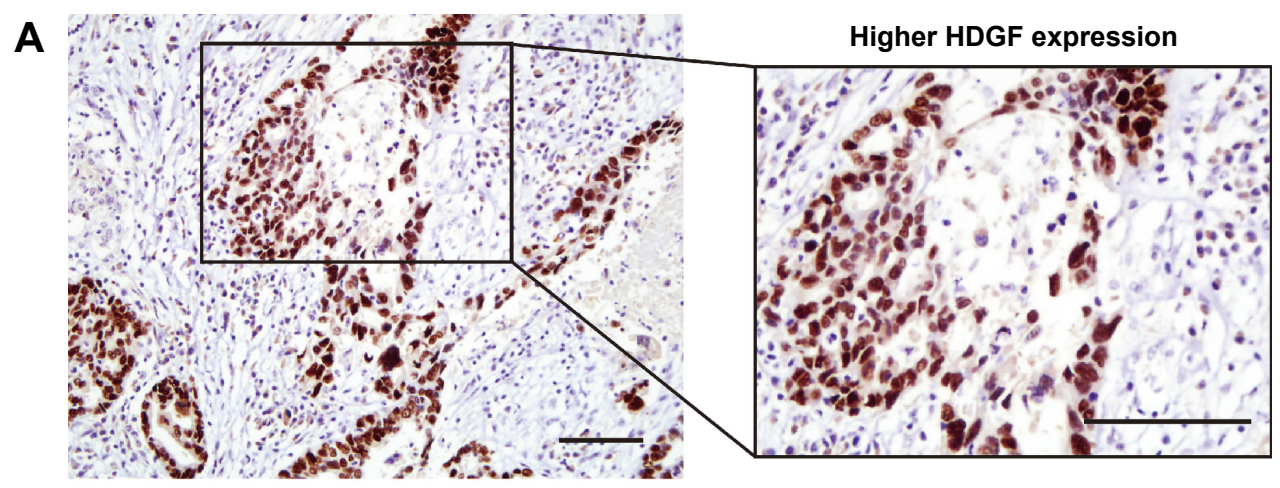

B

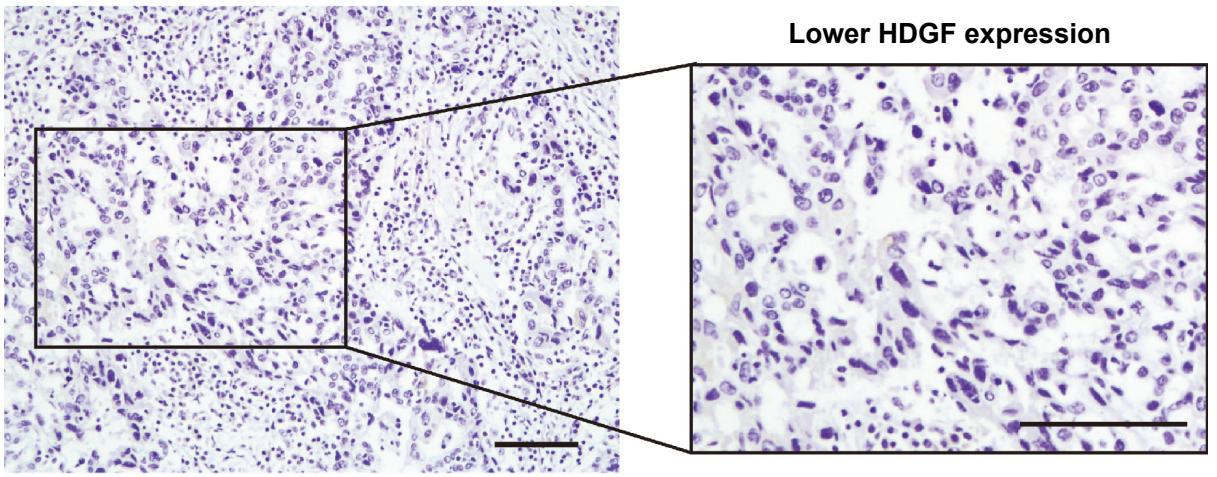

Figure I Representative figures of higher (A) and lower (B) HDGF immunohistochemistry staining in clinical samples of EOC.

Note: Scale bar: $100 \mu \mathrm{m}$

Abbreviations: EOC, epithelial ovarian cancer; HDGF, hepatoma-derived growth factor.

higher-expression was demonstrated to lead to poorer prognosis than HDGF lower-expression $(P=0.003)$. Moreover, in our experiments, higher FIGO stage $(P=0.013)$ and positive lymph node metastasis $(P=0.017)$ were also proved to be significantly associated with the overall prognosis (Figure 2B and C).

Table I Basic characters of patients

\begin{tabular}{lll}
\hline Characteristics & Number & Percentage \\
\hline Age & 26 & \\
$\quad<50$ & 59 & 30.60 \\
$\quad \geq 50$ & & 69.41 \\
FIGO stage & 35 & \\
$\quad$ I-II & 50 & 41.18 \\
$\quad$ III-IV & & 58.82 \\
Histology & 68 & \\
$\quad$ Serous & 17 & 80.00 \\
$\quad$ Non-serous & & 20.00 \\
Lymph node metastasis & 41 & \\
$\quad$ No & 44 & 48.24 \\
Yes & & 51.76 \\
Pathological grade & 18 & \\
I & 37 & 21.18 \\
II & 30 & 43.53 \\
III & & 35.29 \\
HDGF & $4 I$ & 48.24 \\
Low & 44 & 52.76 \\
High
\end{tabular}

Abbreviations: FIGO, International Federation of Gynecology and Obstetrics; HDGF, hepatoma-derived growth factor.

\section{HDGF as an independent prognostic factor in EOC}

The independent prognostic factors were further identified with multivariate analysis (Table 4). The age, FIGO stage, histology, lymph node status, pathological grade, and HDGF

Table 2 Correlation between HDGF expression and clinicopathologic parameters

\begin{tabular}{llll}
\hline Characteristics & \multicolumn{2}{l}{ HDGF } & P* \\
\cline { 2 - 3 } & Low & High & \\
\hline Age & 11 & 15 & 0.529 \\
$\quad<50$ & 30 & 29 & \\
$\quad \geq 50$ & & & \\
FIGO stage & 19 & 16 & 0.350 \\
I-II & 22 & 28 & \\
III-IV & & & \\
Histology & 30 & 38 & 0.127 \\
Serous & 11 & 6 & \\
Non-serous & & & \\
Lymph node metastasis & 26 & 15 & 0.006 \\
No & 15 & 29 & \\
$\quad$ Yes & & & \\
Pathological grade & 11 & 7 & 0.449 \\
I & 16 & 21 & \\
II & 14 & 16 & \\
III & &
\end{tabular}

Note: $* \chi^{2}$ test.

Abbreviations: FIGO, International Federation of Gynecology and Obstetrics; HDGF, hepatoma-derived growth factor. 
Table 3 Correlation between clinicopathologic features and overall survival rate

\begin{tabular}{lll}
\hline Characteristics & 5-year survival rate\% & $\boldsymbol{P}^{*}$ \\
\hline Age & 38 & \\
$\quad<50$ & $31 . \mathrm{I}$ & 0.710 \\
$\quad \geq 50$ & & \\
FIGO stage & 47.8 & 0.013 \\
$\quad$ I-II & 20.1 & \\
$\quad$ III-IV & & \\
Histology & 36.7 & 0.730 \\
$\quad$ Serous & 26 & \\
$\quad$ Non-serous & & \\
Lymph node metastasis & 50.6 & 0.017 \\
$\quad$ No & 13.2 & \\
$\quad$ Yes & & \\
Pathological grade & 28.8 & 0.379 \\
I & 28.8 & \\
II & 44.2 & \\
III & & \\
HDGF & 45.1 & \\
Low & 21.4 & \\
High & &
\end{tabular}

Note: *Log-rank test.

Abbreviations: FIGO, International Federation of Gynecology and Obstetrics; HDGF, hepatoma-derived growth factor.

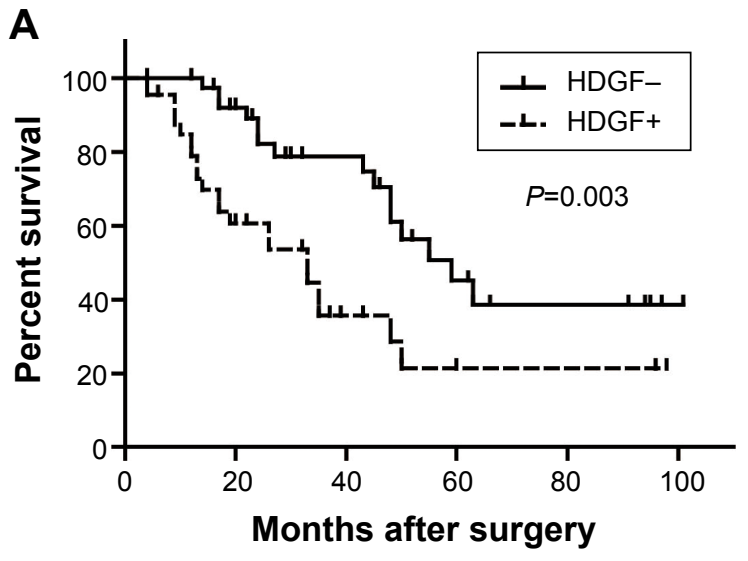

expression were enrolled and analyzed in the Cox-regression hazard model. In the multivariate analysis, HDGF was identified as an independent prognostic factor (hazard ratio $[\mathrm{HR}]=2.80$, confidence interval $[\mathrm{CI}]=1.32-5.95, P=0.007)$. Additionally, pathologic grade II was also proved to have poorer prognosis than grade I and can be considered as a prognostic factor $(\mathrm{HR}=2.59, \mathrm{CI}=1.04-6.42, P=0.040)$.

As lots of previous studies have proved, ${ }^{16-18}$ advanced FIGO stage was also confirmed as an independent prognostic parameter in our system $(\mathrm{HR}=2.73, \mathrm{CI}=1.36-5.53$, $P=0.005)$. Moreover, positive lymph node metastasis tended to be an independent prognostic factor, but this tendency was not statistically significant $(P=0.089)$.

\section{HDGF could promote invasion of EOC cells}

We detected the content of HDGF in EOC cell lines to further confirm the results observed from clinical data. Four ovarian cancer cell lines were collected and selected to detect the HDGF concentration with total cell lysates. HDGF existed

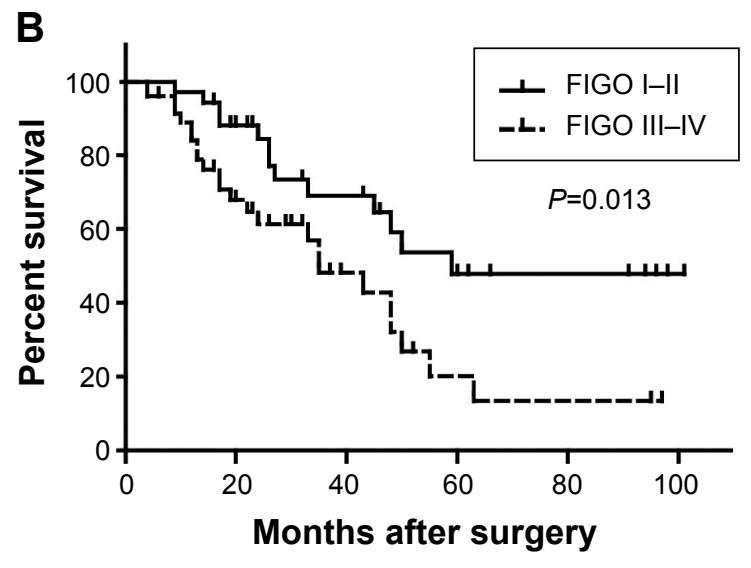

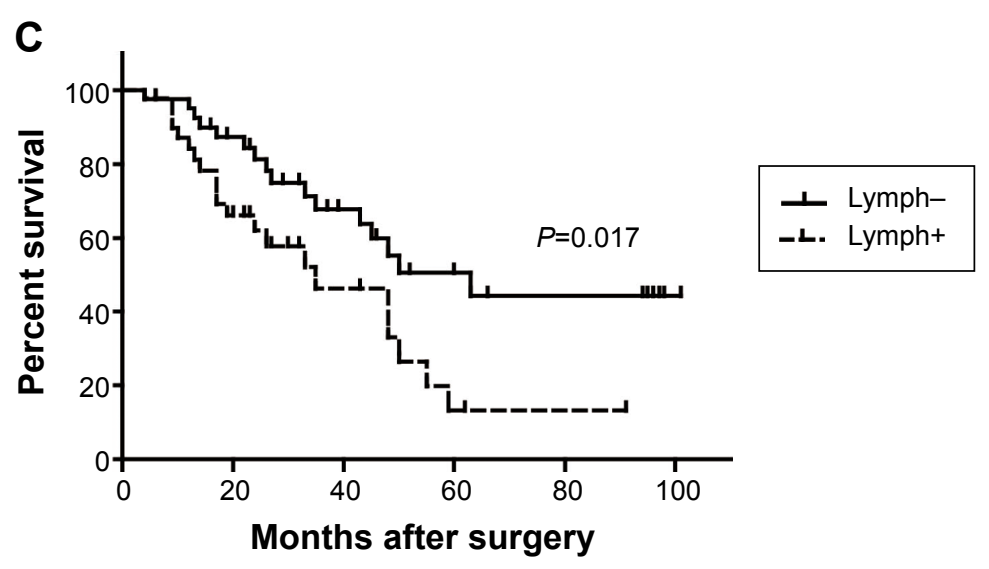

Figure 2 Correlation between HDGF expression, FIGO stage, and lymph node metastasis in validation cohort.

Notes: (A) Higher HDGF predicts unfavorable prognosis of EOC. (B) Advanced FIGO stage is significantly associated with poorer prognosis. (C) Positive lymph node metastasis predicts poorer prognosis.

Abbreviations: EOC, epithelial ovarian cancer; FIGO, International Federation of Gynecology and Obstetrics; HDGF, hepatoma-derived growth factor. 
Table 4 Multivariate analysis

\begin{tabular}{|c|c|c|c|}
\hline Characteristics & HR & $95 \% \mathrm{Cl}$ & $\mathbf{P}^{*}$ \\
\hline \multicolumn{4}{|l|}{ Age } \\
\hline$<50$ & 1 & & \\
\hline$\geq 50$ & 1.23 & $0.58-2.57$ & 0.590 \\
\hline \multicolumn{4}{|l|}{ FIGO stage } \\
\hline I-II & 1 & & \\
\hline III-IV & 2.74 & I.36-5.53 & 0.005 \\
\hline \multicolumn{4}{|l|}{ Histology } \\
\hline Serous & 1 & & \\
\hline Non-serous & 1.4 & $0.61-3.23$ & 0.831 \\
\hline \multicolumn{4}{|c|}{ Lymph node metastasis } \\
\hline No & I & & \\
\hline Yes & 1.9 & $0.91-3.97$ & 0.089 \\
\hline \multicolumn{4}{|l|}{ Pathological grade } \\
\hline 1 & $\mathrm{I}$ & & \\
\hline II & 2.59 & $1.04-6.42$ & 0.040 \\
\hline III & 2 & $0.74-5.35$ & 0.170 \\
\hline \multicolumn{4}{|l|}{ HDGF } \\
\hline Low & 1 & & \\
\hline High & 2.8 & I.32-5.95 & 0.007 \\
\hline
\end{tabular}

Note: *Cox proportional hazards regression.

Abbreviations: $\mathrm{Cl}$, confidence interval; FIGO, International Federation of Gynecology and Obstetrics; HDGF, hepatoma-derived growth factor; HR, hazard ratio.

in all the four cell lines with different expressive rates (Figure 3A). OVCAR3 cells had the highest HDGF expression, while the SK-OV-3 cell line had the lowest HDGF expression. The semiquantitative analysis was achieved by Image-J software and is displayed in Figure 3B, suggesting that OVCAR3 could be a suitable cell model for HDGF knockdown and SK-OV-3 could be the proper HDGF overexpression model. Additionally, we detected the HDGF concentration in the medium of different EOC cell lines (Figure 3C). HDGF was detectable in all these cell lines and the concentration differed along with intracellular HDGF content, indicating that HDGF might be secreted out and could stimulate cell progression in a paracrine way.

In clinical investigation, we observed that HDGF was significantly associated with positive lymph node metastasis, which indicated that HDGF could promote ovarian cancer invasion. So, we performed the transwell assay to explore the role of extracellular recombinant HDGF stimulation in ovarian cell invasion. OVCAR3 or SK-OV-3 cells were passaged into transwell chambers and starved for 6 hours, subsequently incubated in human recombinant HDGF at $10 \mathrm{ng} / \mathrm{mL}$ for 24 hours, followed by fixation with paraformaldehyde, and were stained with gentian violet. In our experiments, exogenous recombinant HDGF could remarkably increase the invasion of OVCAR3 or SK-OV-3 cells, suggesting the essential role of extracellular HDGF in ovarian cancer invasion (Figure 3D). The cellular HDGF significance in invasion was also detected by transwell assay. Successful knockdown by siRNA was first verified by immunoblotting (Figure 3E). Furthermore, the invasive ability of HDGFknocked-down cells was proved to decrease remarkably compared with the cells transfected with scrambled RNA (Figure 3F). This suggests that intracellular HDGF also played an important role in HDGF-induced invasion.

\section{Discussion}

In our study, we detected the expression of HDGF in 85 samples of EOC, and calculated the higher HDGF expression rate as $52.76 \%$. Furthermore, we demonstrated that HDGF expression was significantly associated with lymph metastasis $(P=0.006)$ by $\chi^{2}$ test. With univariate and multivariate analysis, we proved that HDGF expression could be identified as an independent prognostic factor of EOC. Moreover, we demonstrated that HDGF was widely distributed in EOC cell lines with experiments in vitro.

HDGF consists of 240 amino acids, containing an important DNA-binding cassette and several phosphorylation sites. As a transcription factor, HDGF exhibits mitogenic activity for fibroblasts by binding DNA through the N-terminal PWWP domain. ${ }^{19}$ Significance of HDGF in cancer progression and prognosis has been revealed gradually nowadays. HDGF ectopic overexpression has been reported in many kinds of cancers, including gastric cancer, gallbladder cancer, endometrial carcinoma, lung cancer, Ewing's sarcoma, and so on. ${ }^{20-23}$ By regulating HDGF expression in cancer cells or animal models, HDGF was proved to be involved in many processes in cancer cells, including cell transformation, angiogenesis, proliferation, invasion, apoptosis, etc. ${ }^{23} \mathrm{HDGF}$ was usually defined as a survival factor and oncologic trigger. However, the defined signaling pathways and molecular mechanism underlying how HDGF influences cancer progression are still not well elucidated.

HDGF was proved to be associated with the poor prognosis of several kinds of cancers, including cholangiocarcinoma, gallbladder cancer, melanoma, breast cancer, etc. , $20,25^{24}$ In previous studies, HDGF could promote the progression of tumors in many ways, such as accelerating cell growth, increasing cancer stem cells, inducing EMT process, and so on. ${ }^{13,26}$ As a growth factor, the interesting feature of HDGF is that it can be secreted out and activates downstream signaling pathway, which could further promote cancer cell progression. Previous studies showed that HDGF can be regarded as secreted factor in human glioblastoma. Moreover, HDGF can be secreted from tumor cells and stimulate surrounding cells by activating AKT-MAPK signaling pathway or other 
A

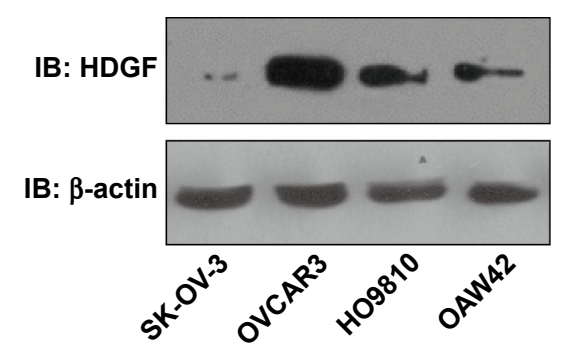

C

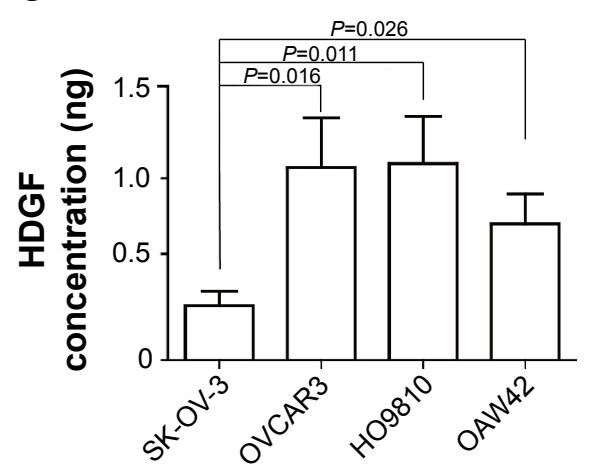

$\mathbf{E}$

IB: HDGF
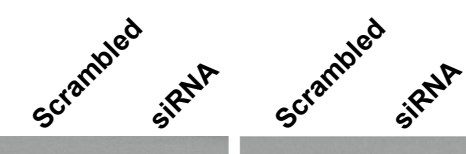

IB: $\beta$-actin

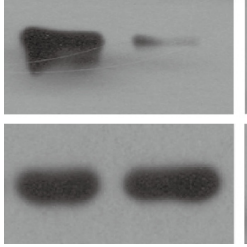

OVCAR3

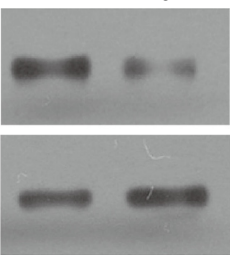

HO9810
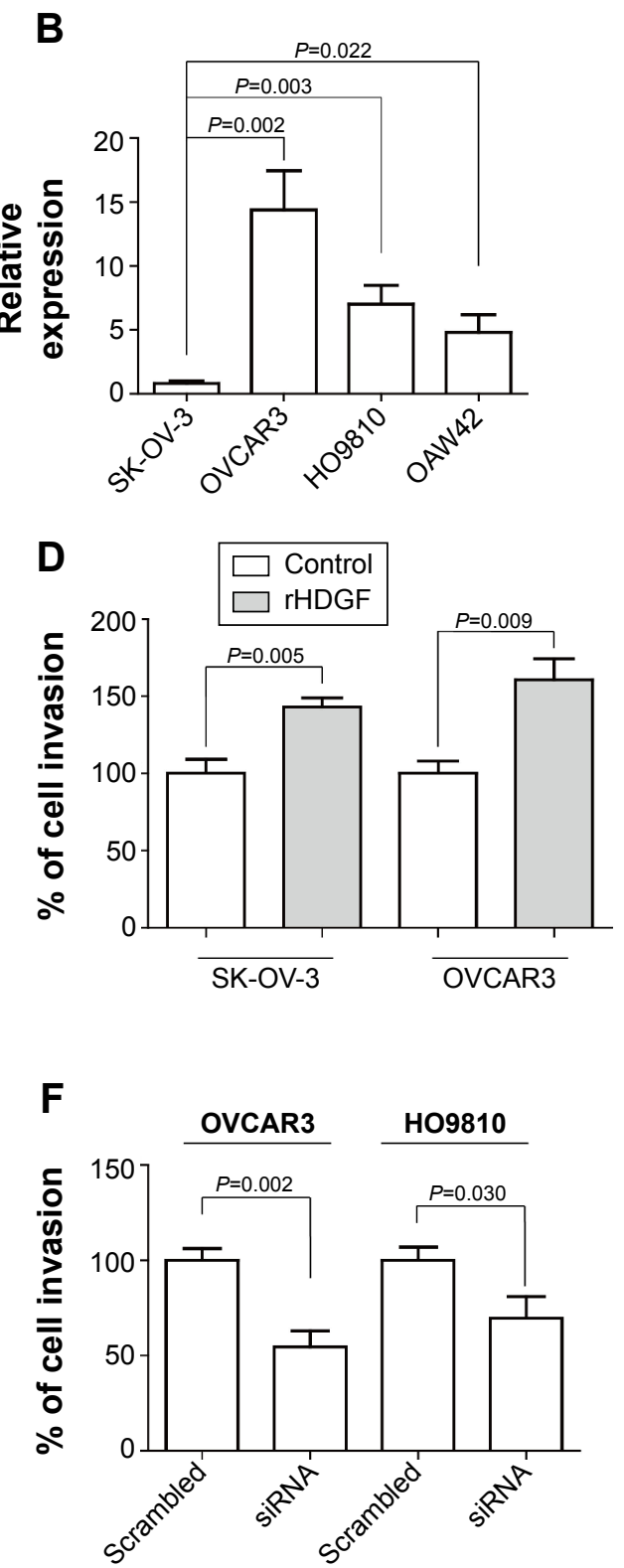

Figure 3 HDGF could promote invasion of EOC cell lines.

Notes: (A) HDGF expression in EOC cell lines SK-OV-3, OVCAR3, HO98I0, and OAW42. (B) Semiquantitation of the result of HDGF expression from immunoblotting was analyzed by Image-J. The statistical significance was evaluated by Student's $t$-test. (C) HDGF concentration in the medium of different EOC cell lines was detected by ELISA. (D) Human recombinant HDGF at $10 \mathrm{ng} / \mathrm{mL}$ could significantly promote the invasion of SK-OV-3 and OVCAR3 cells. Cell numbers were counted and the number of the control group was set as a baseline, with fold change calculated by ratio to the control group. The statistical significance was evaluated by Student's $t$-test. (E) Fortyeight hours after transfection with HDGF siRNA of scrambled RNA, OVCAR3 and HO98I0 cells were lysed and detected by immunoblotting. (F) Invasion of OVCAR3 and HO9810 cells were detected by the transwell method after HDGF was knocked down.

Abbreviations: ELISA, enzyme-linked immunosorbent assay; EOC, epithelial ovarian cancer; HDGF, hepatoma-derived growth factor; IB, immunoblotting; rHDGF, recombinant HDGF.

cell signaling pathways involved in cancer progression. ${ }^{27}$ Therefore, we believed that HDGF may probably function as an oncogenic factor in a paracrine or autocrine way rather than just a survival signal producer.

The main hypotheses from our study involved that HDGF could be secreted from EOC cells, and the increase of HDGF concentration in EOC microenvironment could promote EOC cell progression by a paracrine pathway. In our experiments,
HDGF higher expression was significantly associated with lymphatic invasion, so we highly suspected that HDGF may promote the invasive ability of EOC cells. Considering HDGF as a transcription factor, one important experiment to reveal its underlying function is to find the downstream promoter and protein regulated by HDGF. This mission needs more fundamental experiments to be realized, like microarray after HDGF knockdown, chromatin immunoprecipitation 
assay, luciferase reporter test, etc. Experiments in vivo would be a necessary supply to our study, which may explore underlying mechanism of HDGF-induced EOC progression. However, our study was mainly focused on the clinical significance of HDGF in the prognosis of EOC. We hope our finding that HDGF could be a prognostic biomarker in EOC and can trigger more and more interest among doctors and scientists, finally resulting in the complete elucidation of HDGF's role in EOC.

The main importance of researching a prognostic biomarker is to make it a potential drug target and help find new therapy. Unfortunately, the pharmaceutical value of HDGF has not been discovered. This tarriance is partly due to the fact that the HDGF receptor on the cell surface is unknown. Thakar et $\mathrm{al}^{27}$ reported that HDGF can interact with cell surface with amino acid residues 81-100, but the definite receptor of HDGF has not been found, which makes the discovery of effective HDGF inhibitors hard to achieve. As a potential chemotherapy drug target, our group suggested that more attention should be focused on searching for a HDGF receptor and specific inhibitor, which may help find out a new therapy and improve EOC patients' survival time, like what Herceptin does to breast cancer patients.

\section{Conclusion}

In conclusion, we found that HDGF was widely distributed in EOC and EOC cell lines. HDGF expression was significantly correlated with overall survival rate and could be regarded as an independent prognostic factor, indicating that HDGF could be a potential drug target and could help find new chemotherapy for EOC patients.

\section{Disclosure}

The authors report no conflicts of interest in this work.

\section{References}

1. Partridge EE, Barnes MN. Epithelial ovarian cancer: prevention, diagnosis, and treatment. CA Cancer J Clin. 1999;49:297-320.

2. Lozano R, Naghavi M, Foreman K, et al. Global and regional mortality from 235 causes of death for 20 age groups in 1990 and 2010: a systematic analysis for the Global Burden of Disease Study 2010. Lancet. 2012; 380:2095-2128.

3. Goff BA, Mandel L, Muntz HG, Melancon CH. Ovarian carcinoma diagnosis. Cancer. 2000;89:2068-2075.

4. Barnes MN, Grizzle WE, Grubbs CJ, Partridge EE. Paradigms for primary prevention of ovarian carcinoma. CA Cancer J Clin. 2002;52: 216-225.

5. Barber HR. Ovarian cancer. CA Cancer J Clin. 1986;36:149-184.

6. Basu M, Mukhopadhyay S, Chatterjee U, Roy SS. FGF16 promotes invasive behavior of SKOV-3 ovarian cancer cells through activation of mitogen-activated protein kinase (MAPK) signaling pathway. $J$ Biol Chem. 2014;289:1415-1428.
7. McGuire WP, Hoskins WJ, Brady MF, et al. Cyclophosphamide and cisplatin compared with paclitaxel and cisplatin in patients with stage III and stage IV ovarian cancer. $N$ Engl J Med. 1996;334:1-6.

8. Nakamura H, Izumoto Y, Kambe H, et al. Molecular cloning of complementary DNA for a novel human hepatoma-derived growth factor. Its homology with high mobility group-1 protein. J Biol Chem. 1994;269: 25143-25149.

9. Enomoto H, Yoshida K, Kishima Y, et al. Hepatoma-derived growth factor is highly expressed in developing liver and promotes fetal hepatocyte proliferation. Hepatology. 2002;36:1519-1527.

10. Chang KC, Tai MH, Lin JW, et al. Hepatoma-derived growth factor is a novel prognostic factor for gastrointestinal stromal tumors. Int $J$ Cancer. 2007;121:1059-1065.

11. Uyama H, Tomita $\mathrm{Y}$, Nakamura $\mathrm{H}$, et al. Hepatoma-derived growth factor is a novel prognostic factor for patients with pancreatic cancer. Clin Cancer Res. 2006;12:6043-6048.

12. Yamamoto S, Tomita Y, Hoshida Y, et al. Expression of hepatomaderived growth factor is correlated with lymph node metastasis and prognosis of gastric carcinoma. Clin Cancer Res. 2006;12:117-122.

13. Kishima Y, Yamamoto H, Izumoto Y, et al. Hepatoma-derived growth factor stimulates cell growth after translocation to the nucleus by nuclear localization signals. J Biol Chem. 2002;277:10315-10322.

14. Liu YF, Zhao R, Guo S, et al. Expression and clinical significance of hepatoma-derived growth factor as a prognostic factor in human hilar cholangiocarcinoma. Ann Surg Oncol. 2011;18:872-879.

15. Guo Z, He Y, Wang S, et al. Various effects of hepatoma-derived growth factor on cell growth, migration and invasion of breast cancer and prostate cancer cells. Oncol Rep. 2011;26:511-517.

16. Anttila M, Kosma VM, Ji H, et al. Clinical significance of alpha-catenin, collagen IV, and Ki-67 expression in epithelial ovarian cancer. J Clin Oncol. 1998;16:2591-2600.

17. Shen Y, Li DD, Wang LL, Deng R, Zhu XF. Decreased expression of autophagy-related proteins in malignant epithelial ovarian cancer. Autophagy. 2008;4:1067-1068.

18. Vergote I, De Brabanter J, Fyles A, et al. Prognostic importance of degree of differentiation and cyst rupture in stage I invasive epithelial ovarian carcinoma. Lancet. 2001;357:176-182.

19. Yang J, Everett AD. Hepatoma-derived growth factor binds DNA through the N-terminal PWWP domain. BMC Mol Biol. 2007;8:101.

20. Tao F, Ye MF, Sun AJ, et al. Prognostic significance of nuclear hepatoma-derived growth factor expression in gallbladder cancer. World J Gastroenterol. 2014;20:9564-9569.

21. Wang L, Jiang Q, Hua S, et al. High nuclear expression of HDGF correlates with disease progression and poor prognosis in human endometrial carcinoma. Dis Markers. 2014;2014:298795.

22. Chen B, Huang T, Jiang J, Lv L, Li H, Xia S. miR-141 suppresses proliferation and motility of gastric cancer cells by targeting HDGF. Mol Cell Biochem. 2014;388:211-218.

23. Bao C, Wang J, Ma W, Wang X, Cheng Y. HDGF: a novel jack-of-alltrades in cancer. Future Oncol. 2014;10(16):2675-2685.

24. Chen X, Yun J, Fei F, et al. Prognostic value of nuclear hepatomaderived growth factor (HDGF) localization in patients with breast cancer. Pathol Res Pract. 2012;208:437-443.

25. Tsai HE, Wu JC, Kung ML, et al. Up-regulation of hepatoma-derived growth factor facilitates tumor progression in malignant melanoma [corrected]. PloS One. 2013;8:e59345.

26. Zhao J, Ma MZ, Ren H, et al. Anti-HDGF targets cancer and cancer stromal stem cells resistant to chemotherapy. Clin Cancer Res. 2013;19: 3567-3576.

27. Thakar K, Kröcher T, Savant S, Gollnast D, Kelm S, Dietz F. Secretion of hepatoma-derived growth factor is regulated by n-terminal processing. Biol Chem. 2010;391:1401-1410. 
OncoTargets and Therapy

\section{Publish your work in this journal}

OncoTargets and Therapy is an international, peer-reviewed, open access journal focusing on the pathological basis of all cancers, potential targets for therapy and treatment protocols employed to improve the management of cancer patients. The journal also focuses on the impact of management programs and new therapeutic agents and protocols on

patient perspectives such as quality of life, adherence and satisfaction. The manuscript management system is completely online and includes a very quick and fair peer-review system, which is all easy to use. Visit http://www.dovepress.com/testimonials.php to read real quotes from published authors.

Submit your manuscript here: http://www.dovepress.com/oncotargets-and-therapy-journal 